\title{
Study on the Estrous Cycle Regularity of Cryopreserved Rat Ovarian Tissues after Heterotopic Transplantation
}

\author{
Rumana Jafarey ${ }^{*}$, Syed Ali Rehan Shah Jaffri² \\ ${ }^{1}$ Reproductive Medical Centre, Renmin Hospital of Wuhan University, Wuhan, China \\ ${ }^{2}$ Federal Board of Revenue, Islamabad, Pakistan \\ Email: "doccr2003@yahoo.com
}

Received 15 March 2016; accepted 23 April 2016; published 26 April 2016

Copyright (C) 2016 by authors and Scientific Research Publishing Inc.

This work is licensed under the Creative Commons Attribution International License (CC BY). http://creativecommons.org/licenses/by/4.0/

(c) (i) Open Access

\begin{abstract}
Objective: To evaluate the re-initiation of ovarian function in cryopreserved ovarian grafts by means of vaginal smear of transplant rats. Methods: A total of 40 SPF-SD female rats (5 - 6 week-old) were randomly divided into three groups (blank control, castration control and transplant group). Ovaries were removed by surgical procedure then after cryopreservation and thawing procedures the ovarian tissues pushed inside the back muscles gap in transplant group. On the first PO day, vaginal smear collection was daily initiated. After 30 days, the PO day when the estrous cycle was re-initiated was considered for analysis as well as the estrous days and the number of estrous cycles. Results: Normal control group had a regular estrous cycle, while the transplant group had an estrous cycle disorder within first 2 weeks and later 2 weeks after the transplantation while the duration of diestrus cycle lasted longer. At the same time, the castration group had lost the normal estrous cycle, keeping continue in the stage of diestrus. Conclusion: In transplanted animals the re-initiated ovarian function can be predicted with alteration between estrous and diestrus phases with predominant estrous irregularity. Moreover, short autotransplanted graft duration needs time to perfuse by new blood vessels and hormone secretions, so could not directly affect its target organs to function properly.
\end{abstract}

\section{Keywords}

Transplantation, Cryopreservation, Thawing, Vaginal Smear, Ovarian Tissue, Rats

\section{Introduction}

In the last ten years, the number of long-term cancer survivors has increased markedly by the rapid growth of

${ }^{*}$ Corresponding author.

How to cite this paper: Jafarey, R. and Jaffri, S.A.R.S. (2016) Study on the Estrous Cycle Regularity of Cryopreserved Rat Ovarian Tissues after Heterotopic Transplantation. Open Journal of Obstetrics and Gynecology, 6, 293-298.

http://dx.doi.org/10.4236/ojog.2016.65037 
robust technologies for fertility preservation in female cancer patients [1]-[4].

The subsequent transplantation is considered as an alternative treatment to restore fertility in cancer female patients who are at risk of ovarian failure after deleterious effects of cancer therapy [2] [4] [5].

In order to maintain the reproductive capacity of cancer female patients, the cryopreserved ovarian transplant (orthotopic or heterotopic site) is considered as the most promising one method due to the presence of large number of primordial follicles which are potentially capable to mature and more resistant to aggression by transplantation and cryopreservation process [3] [5]-[7]. While earlier studies have considered that the major loss of viable follicles quantity occurred after transplantation rather than freeze-thaw procedure because transplantation directly related to time duration for re-establishing of blood supply to graft tissues [5] [8].

The longer the time course of angiogenesis, the shorter is graft survival so measures to accelerate revascularization are greatly expected by specialists [6]. Many studies have shown that despite a drastic reduction in primordial follicle number, cyclical ovarian function can be restored after fresh or cryopreserved heterotopic ovarian transplantation [9]-[11]. In rodents, the study of vaginal cytological smear is an efficient method in the evaluation of ovarian function due to the short life of the estrous cycle as well as the facility to characterize its many phases [5] [6].

The characterization of each phase is based on the proportion among three types of cells observed in vaginal smear: epithelial cells, cornified cells and leukocytes [1]. The median duration of the estrous cycle is 4 to 5 days in $60 \%$ to $70 \%$ of the animals. Irregular cycles are characterized when phase alternation does not present a pro estrous, metaestrous and diestrous sequence or when there is permanence in the same phase for four to five days. Some experimental studies use vaginal smear cytological analysis as an indirect and dynamic parameter of ovarian activity re-initiation, after the transplant [5].

The vaginal smear cytological analysis has long been employed as a relatively noninvasive method to document reproductive cycles in laboratory rats and mice and to provide an index of functional status of hypothalamic-pituitary ovarian axis. A few years after the early $20^{\text {th }}$ century discovery by Stockard and Papanicolaou of rhythmic changes in the appearance of epithelial cells with Guinae pig vaginal lumen, this method has been used both as a principal measure in laboratory determinations of reproductive cyclicity and as an ancillary component of studies in Reproductive Toxicology [12].

Despite of more progress in vaginal smear cytological analysis technique after heterotopic transplantation for evaluation of estrous cycle re-initiation in laboratory rodent, is still in experimental stage and further research is necessary to characterize stages of the reproductive cycle, to determine the loss of a considerable quantity of viable follicles and ovarian activity re-initiation after orthotopic or heterotopic transplantation.

Therefore this present study focuses on the the estrous cycle regularity of cryopreserved rat ovarian tissues after heterotopic transplantation.

\section{Materials and Methods}

\subsection{Animals}

The experiments were carried out using 40 SPF-SD female rats, aged between ( 5 - 6 weeks-old) weighing between 200 and 250 gm, purchased from the Hunan Silai Kejing Da Experimental Animal Co., Ltd., China. All animals were cared for and used in accordance with International Guiding Principle for Biomedical Research Involving Animals at Renmin Hospital of Wuhan University Experimental Animal house, China. They were housed under a lighting regimen of 12 hour light and 12 hour darkness and temperature-controlled conditions $\left(22^{\circ} \mathrm{C} \pm\right.$ $2^{\circ} \mathrm{C}$ ). Animals were fed a standard laboratory animal feed (Beijing Bio-Technology Co., Ltd.) and water.

\subsection{Experimental Design}

The animals were randomly divided into three groups. (a) Blank control group $n=15$ : no treatment (b) Castrated control group $n=15$ : ovariectomy (c) Transplant group $n=10$ : transplantation of cryopreserved-thawed ovarian tissues into back muscles.

\subsection{Bilateral Ovariectomy}

The rats were anesthetized by $10 \%$ Chloral Hydrate $(0.3 \mathrm{ml} / 100 \mathrm{gm})$ i.p injection. All the following surgical procedures were performed aseptically. A single median longitudinal skin incision was made on the lumbar por- 
tion to expose the subcutaneous tissue over the ovary. In group B, bilateral ovariectomy was performed without transplantation as these rats served as a castrated control group. While in group C, a small incision was made on the fascia and muscles immediately above the ovary, thereby exteriorizing the reproductive tract, to isolate and resects both ovaries surgically. In the end of surgery rats were injected with 16,000 IU of penicillin in $1 \mathrm{ml} 0.9 \%$ $\mathrm{NaCl}$ by intraperitoneal.

\subsection{Cryopreservation and Thawing}

The recovered ovaries of group $\mathrm{C}$ were transported immediately in cell culture dish (Corning) containing $2 \mathrm{ml}$ [HTF medium (M\&C Gene Technology)] and then ovarian cortex was processed at room temperature $\left(25^{\circ} \mathrm{C}\right)$ and cut into small sections (between $2 \times 2 \times 3 \times 3 \mathrm{~mm}$ in size). Ovarian cortical sections were transferred into cell culture dishes in stepwise manners, containing solution 1 [15\% EG + 15\% DMSO (Sigma-Aldrich) + 0.5 M sucrose (Sigma-Aldrich) with $10 \mathrm{ml}$ HTF-HEPES (In Vitro Care, Frederick, USA)] remained for 30 - 45 seconds, then transferred in solution 2 (7.5\%EG + 7.5\%DMSO) remained for 3 minutes and finally transferred in solution 3 (12\% HTF-HEPES) remained for 3 minutes at room temperature. The ovarian tissue were then transferred in cryogenic vials and plunged into liquid nitrogen for storage.After the storage time for 21 days in liquid nitrogen, the cryopreserved ovarian tissue were thawed rapidly $\left(\sim 100^{\circ} \mathrm{C} / \mathrm{min}\right)$ by agitating in a warm water bath $\left(35^{\circ} \mathrm{C}\right)$ for 2 to 3 minutes and washed in a stepwise manner to minimize osmotic damages $(1.0 \mathrm{M}$ sucrose $+10 \mathrm{ml}$ HTF-HEPES for one minute, $0.5 \mathrm{M}$ sucrose $+10 \mathrm{ml}$ HTF-HEPES for 3 minute and 12\% HTF-HEPES for 3 minutes) at room temperature prior to transplantation.

\subsection{Transplantation Procedure}

The rats were anesthetized by $10 \%$ Chloral Hydrate $(0.3 \mathrm{ml} / 100 \mathrm{gm})$ i.p injection. All the following surgical procedures were performed aseptically. The back skin and muscle was cut and thawed ovarian tissues were immediately inserted inside the muscle gap (made with ophthalmologic tweezers) in group $\mathrm{C}$ and then the incision was closed with stitches of nylon sutures. In the end of surgery rats were injected with 16,000 IU of penicillin in $1 \mathrm{ml} 0.9 \% \mathrm{NaCl}$ by intraperitoneal.

\subsection{Vaginal Smear Collection}

On the first postoperative day (the nest day of transplantation), every morning between 8:00 and 9:00 a.m. vaginal smear collection was daily reinitiated, for 30 days. Vaginal smear collection was performed (the animals were kept immobile) with a swab imbibed in $250 \mathrm{ml}$ of normal saline ( $\mathrm{NaCl} 0.9 \%)$. Vaginal fluid was placed on standard glass slides. A different glass slide was used for each cage of three group animals. Unstained material was observed under a light microscope, without the use of the condenser lens, with $10 \times$ and $40 \times$ objective lenses. The following phases of the estrous cycle were considered, according to the proportion of observed cells in the smears: 1) Proestrous phase: Predominance of epithelial nuclear cells; 2) estrous phase: Predominance of keratinized anucleate cells; 3) diestrous phase: The same proportion of leukocytes and nuclear epithelial and keratinized cells.

\subsection{Statistical Analysis}

PASW Statistics software (version 18.0) was used for data evaluation. For the analysis of the results, Variance Analysis (ANOVA) was used to compare values across estrous stage during first 2 weeks and later 2 weeks post transplantation period.

\section{Results}

\subsection{The Estrous Cyclicity of Rats in Four Weeks after Transplantation}

Vaginal smears were taken every other day to detect the estrous cyclicity of the rats after the transplantation.

\subsubsection{The Vaginal Smear Observation in Different Stages of Estrous Cyclicity}

Figure 1 indicates the cellular types from the vaginal smear of female rats, and the proportion among them allows the observer to define the estrous cycle phase of the rat after heterotopic transplantation. A protestrus 
smear consists of a predominance of nucleated epithelial cells; an estrous smear primarily consists of anucleated cornified cells; a metestrus smear consists of the same proportion among leukocytes, cornified, and nucleated epithelial cells, and a diestrus smear primarily consists of a predominance of leuokocytes.

\subsubsection{The Changes of Estrous Cyclicity in Four Weeks after Transplantation}

Figure 2 indicates that the normal control group had a regular estrous cycle, while the transplant group had an

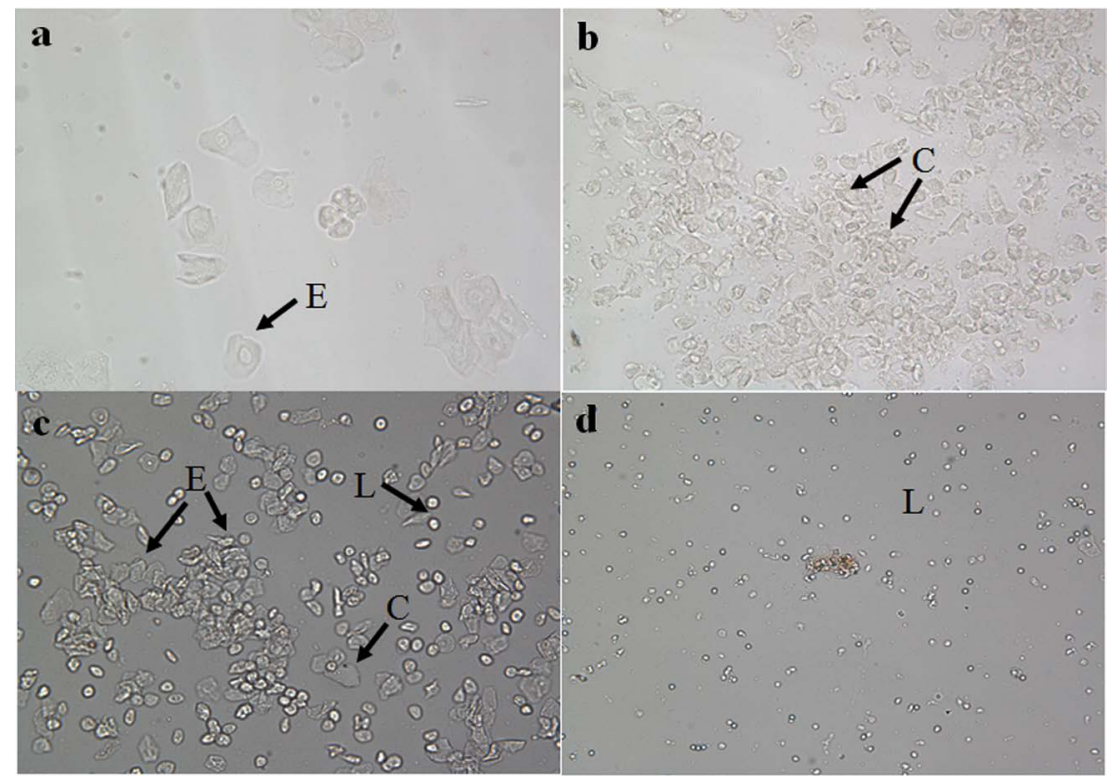

Figure 1. Photomicrographs of unstained vaginal smear from female rats (a) a proestrus smear consists of a predominance of nucleated epithelial cells (E); (b) an estrous smear primarily consists of anucleated cornified cells (C); (c) a metestrus smear consists of the same proportion among leukocytes (L), cornified (C), and nucleated epithelial cells (E); (d) a diestrus smear primarily consists of a predominance of leukocytes $(\mathrm{L})$.

(a)

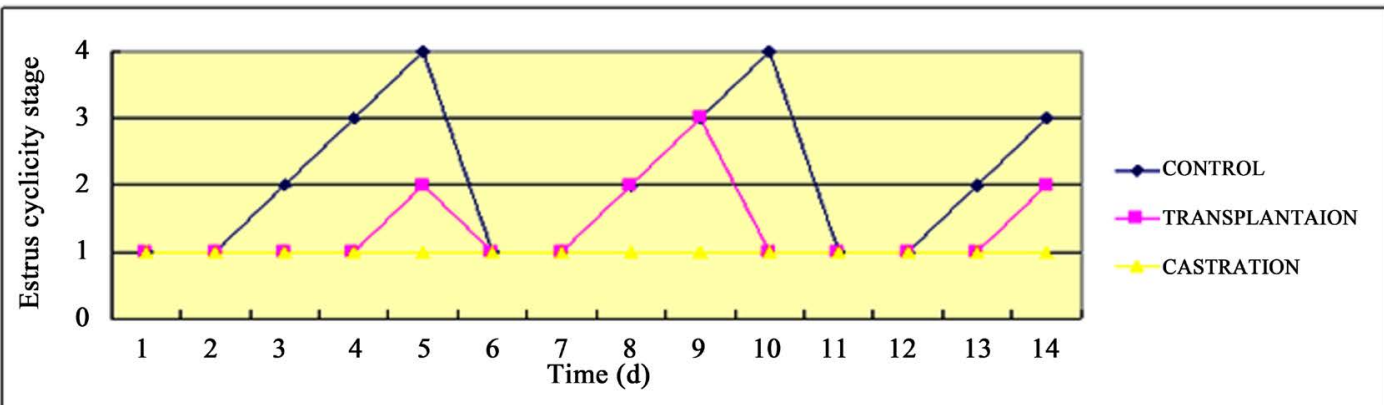

(b)

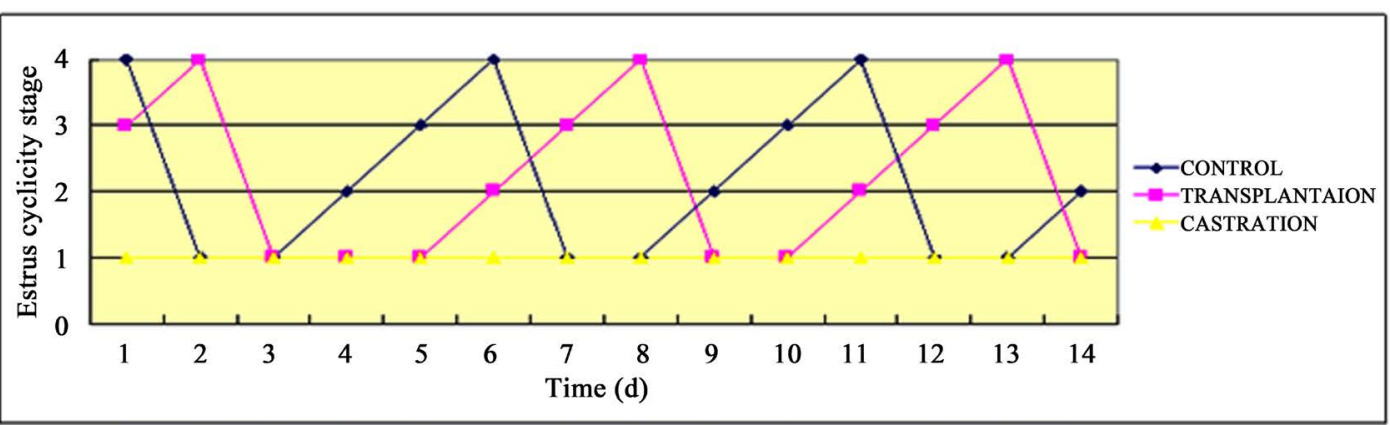

Figure 2. Days of estrous re-initiation. (a) First 2 week after transplantation; (b) later 2 weeks after transplantation. (Stages: 1 = Diestrus, 2 = Proestrus, 3 = Estrous, 4 = Metestrus). 
estrous cycle disorder within first 2 weeks and later 2 weeks after the transplantation while the duration of diestrus cycle lasted longer. At the same time, the castration group lost the normal estrous cycle, keeping continues in the stage of diestrus.

\section{Discussion}

In recent years, it has been most obvious that the site of grafting is very important, since it influences tissue survival and follicle development as well as the quality of the obtained oocytes after transplantation.Vaginal cytology is a simple technique that can be used by practitioners to help to characterize stages of the reproductive cycle to evaluate certain diseases of the genital tract. Generally estrous cycle in rats and mice averages 4 - 5 days and is divided into four stages including (proestrus, estrus, metestrus and diestrus).

Proestrus is a short stage which immediately proceeds to estrus phase, lasting an average of 14 days in rats and less than 24 hours in mice. The predominate feature of this stage is presence of small, round and nucleated epithelial cells, increasing of estradiol concentration, vaginal epithelial proliferation accompanied by erythrocytes diapedesis from uterine capillaries thereby most of the erythrocytes observed on vaginal smear at this reproductive stage. Grossly, vulvar edema, hyperemia, and a serosanguineous discharge occur.

Estrus duration ranges between 24 and 48 hours in rats and between 12 and 48 hours in mice. This is characterized by presence of predominately anucleated keratinized epithelial cells and considered as the period of copulation. During estrus phase the glandular secretions increase, the vaginal epithelium becomes hyperemic and ovulation occurs. This reproductive cycle stage is mainly influenced by estrogens. The interval between successive estrus cycles is typically seven months.

Metestrus is considered as a short stage of 6 - 8 hours in rats while in mice last up to 24 hours and presence of a combination of anucleated keratinized epithelial cells and neutrophils. In early metestrus the neutrophils combined with epithelial cells in same proportion, while in late metestrus the neutrophils and epithelial cell numbers decrease with decrease in smear cellularity before progress to diestrus phase.

Diestrous is the longest period with an average of 48 to 72 hours in both mice and rats. This phase is mainly characterized by the activity of corpus luteum and a significant reduction in the number of anucleated keratinized epithelial cells. During this phase, the corpus luteum is fully functional and produces progesterone. Physiologically, the estrogen stimulation signs subside and uterine glands undergo hypertrophy and hyperplasia. The cervix constricts and vaginal secretions become tenacious. In absence of pregnancy the diestrus phase terminates with the regression of corpus luteum which takes an average of 70 - 80 days.

In the mouse model, heterotopic ovarian transplantation sites such as beneath the kidney capsule and inside the back muscle have also been explored. Higher survival rate of primordial follicles and better vascularisation after transplantation of fresh hemiovaries into the back muscle was seen as compared to under the kidney [5] [13] [14]. The method that has been in the recent studied in order to preserve female reproductive function is the cryopreserved ovarian transplant either at an orthotopic or heterotopic site, due to the presence of a large number of primordial follicles potentially capable of maturing. These follicles are more resistant not only to aggression has result from the transplant but also from the cryopreservation process, which makes this technique a very promising one.

The transplantation itself is the principal factor contributing to the loss of a considerable quantity of viable follicles and is directly related to the necessary time for the re-establishing of blood supply and consequently compromising graft survival. The longer the time course of angiogenesis, the shorter is graft survival. Thus, measures to accelerate revascularization are greatly expected by specialists.

In rodents, the study of vaginal cytological smear is an efficient method in the evaluation of ovarian function due to the short life of the estrous cycle as well as the facility to characterize its many phases. The median duration of the estrous cycle is 4 to 5 days in $60 \%$ to $70 \%$ of the animals. Irregular cycles are characterized when phase alternation does not present a proestrous - metestrous - diestrous sequence or when there is permanence in the same phase for four to five days. Some experimental studies use vaginal smear cytological analysis as an indirect and dynamic parameter of ovarian activity re-initiation, after the transplant [14].

In our study, estrous cyclicity variation was observed with diestrus long duration within 2 weeks period after transplantation of cryopreserved ovarian tissue in transplanted and castration group respectively. Normally the ovarian function in the graft re-initiate if that establish angiogenesis at the site of auto transplantation and once vascular web established, this may stimulate the graft to re-initiate its function which can be justifies by findings in the vaginal cytology smears. In transplanted animals the re-initiated ovarian function can be predicted with 
alteration between estrous and diestorus phases with predominate estrous irregularity [13]. In addition the perfused transplanted grafts secrete hormones and affect on its target organs through the new established vessels [6]. Functional vessels within grafts have been detected in rats by third 3 day.

In our results, transplanted group showed predominate irregular estrous phase with diestrous long duration phase which may be due to graft hyper-stimulation as an adaptation to the new implant site which lead to shortening of the pro-estrous phase of ovulation. Moreover, our autotransplanted graft duration was for short duration ( 2 weeks and 4 weeks), due to which the graft ovarian tissue might have taken time to perfuse with new blood vessels and could not generate the growth hormones include-GnRH (which could stimulate the anterior pituitary to secrete FSH or LH to positively affect on ovaries), so could not directly affect the ovulation.

\section{Conclusion}

The ovarian function in the graft re-initiate if that establish angiogenesis at the site of auto transplantation and once vascular web established, this may stimulate the graft to re-initiate its function which can be justified by findings in the vaginal cytology smears. In transplanted animals the re-initiated ovarian function can be predicted with alteration between estrous and diestorus phases with predominate estrous irregularity. Moreover, short autotransplanted graft duration needs time to perfuse by new blood vessels and hormone secretions, so could not directly affect its target organs to function properly.

\section{References}

[1] Marcondes, F.K., Bianchi, F.J. and Tanno, A.P. (2002) Determination of the Estrous Cyclephases of Rats: Some Helpful Considerations. Brazilian Journal of Biology, 62, 609-614. http://dx.doi.org/10.1590/S1519-69842002000400008

[2] Kim, S.S., Lee, W.S., Chung, M.K., Lee, H.C., Lee, H.H. and Hill, D. (2009) Long-Term Ovarian Function and Fertility after Heterotopic Autotransplantation of Cryobanked Human Ovarian Tissue: 8-Year Experienced in Cancer Patients. Fertility and Sterility, 91, 2349-2354. http://dx.doi.org/10.1016/j.fertnstert.2008.04.019

[3] Jafarey, R. and Yang, J. (2012) Morphological Ultrastructural Changes in Rat Ovarian Tissues after Cryopreservation. Chinese-German Journal of Clinical Oncology, 11, 527-532. http://dx.doi.org/10.1007/s10330-012-1026-5

[4] Donnez, J., Dolmans, M., Demylle, D., Jadoul, P., Pirard, C., Squifflet, J., Martinez, M.B. and Van, L.A. (2004) Livebirth after Orthotopic Transplantation of Cryopreserved Ovarian Tissue. Lancet, 364, 1405-1410. http://dx.doi.org/10.1016/S0140-6736(04)17222-X

[5] Damous, L.L., Silva, S.M., Lopes, R.A.M., Sakano, C.R.S.B., Simoes, M.J. and Montero, E.F.S. (2009) Study on the Vaginal Smear of Rats Submitted to Autologus Ovarian Transplant. Impact of Remlote Ischemic Preconditioning. Acta Cirurgica Brasileris, 24, 387-92. http://dx.doi.org/10.1590/S0102-86502009000500009

[6] Bedaiwy, M.A., El-Nashar, S.A., ElSaman, A.M., Evers, J.L.H., Sandadi, S., Desai, N. and Falcone, T. (2008) Reproductive Outcome after Transplantation of Ovarian Tissue: A Systemic Review. Human Reproduction, 23, 2709-2717. http://dx.doi.org/10.1093/humrep/den301

[7] Boldt, J., Cline, D. and McLaughlin, D. (2003) Human Oocyte Cryppreservation as an Adjunct to IVF-Embryo Transfer Cycles. Human Reproduction, 18, 1250-1255. http://dx.doi.org/10.1093/humrep/deg242

[8] Liu, J., Elst, J.V., Broecke, R.V. and Dhont, M. (2002) Early Massive Follicle Loss and Apoptosis in Heterotopically Grafted Newborn Mouse Ovaries. Human Reproduction, 17, 605-611. http://dx.doi.org/10.1093/humrep/17.3.605

[9] Torrents, E., Boiso, I., Barri, P.N. and Veiga, A. (2003) Applications of Ovarian Tissue Transplantation in Experimental Biology and Medicine. Human Reproduction, 19, 471-481. http://dx.doi.org/10.1093/humupd/dmg036

[10] Braid, D.T., Webb, R., Campbell, B.K., Harkness, L.M. and Gosden, R.G. (1999) Long Term Ovarian Function in Sheep after Ovariectomy and Transplantation of Autografts Stored at $-196^{\circ}$ C. Endocrinology, 140, 462-471.

[11] Callejo, J., Salvador, C., Miralles, A., Vilaseca, S., Lailla, J.M. and Balasch, J. (2001) Long Term Ovarian Function Evaluation after Autografting by Implantation with Fresh and Frozen Thawed Human Ovarian Tissue. The Journal of Clinical Endocrinology \& Metabolism, 86, 4489-4494. http://dx.doi.org/10.1210/jcem.86.9.7871

[12] Goldman, J.M., Murr, A.S. and Cooper, R.L. (2007) The Rodent Estrous Cycle: Characterization of Vaginal Cytology and Its Utility in Toxicology Studies. Birth Defects Research. Part B, Developmental and Reproductive Toxicology, 80, 84-97. http://dx.doi.org/10.1002/bdrb.20106

[13] Milenkovic, M. (2011) Experimental Studies on Ovarian Cryopreservation and Transplantation. Geson Hylte Goteborg Press, Sweden, 1-118.

[14] Cora, M.C., Kooistra, L. and Travlos, G. (2015) Vaginal Cytology of the Laboratory Rat and Mouse: Review and Criteria for the Staging of the Estrous Cycle Using Stained Vaginal Smears. Toxicologic Pathology, 1-18. 